\title{
HIPOGONADISMO ASOCIADO A LA SENILIDAD EN EL VARÓN (CLIMATERIO MASCULINO - ANDROPAUSIA - ADAM)
}

\author{
Enzo Devoto C. ${ }^{1}$, Lucía Aravena C. ${ }^{1}$ \\ 1 Facultad de Medicina, Universidad de Chile, Campus Central, Servicio de Medicina, Unidad de Endocrinología, \\ Hospital Clínico San Borja - Arriarán.
}

\section{RESUMEN}

Actualmente se acepta que en hombres envejecidos sanos existe un descenso progresivo aunque no universal, de la testosterona total y libre, llegando un 20 a $30 \%$ a rango hipogonádico. El diagnóstico de hipogonadismo asociado a envejecimiento es de exclusión. Se requieren estudios para poder diferenciar si los síntomas compatibles con hipogonadismo se deben a falla testosterónica, envejecimiento o alteración de otros ejes endocrinos.

\section{PALABRAS CLAVES: Andropausia, hipogonadismo, testosterona, envejecimiento, función testicular}

\section{SUMMARY}

At present it is accepted that exist in healthy aged men a progressive reduction of the total and free testosterone, arriving $20-30 \%$ to hypogonadism level. The diagnosis of associated hypogonadism to aging is of exclusion. More studies are required to differentiate if the compatible symptoms with hypogonadism must be to testosterone deficiency, aging or alteration of other endocrine axes.

KEY WORDS: Andropause, ADAM, hypogonadism, testosterone, aging, testicular function

\section{INTRODUCCIÓN}

No existe la suficiente conciencia a nivel médico de estudiar el hipotestosteronismo (hipogonadismo) en el varón y menos aún en el envejecido; un gran desconocimiento ocurre a nivel del público respecto a este tema, de sus síntomas y de la posibilidad de reemplazo androgénico. La presente revisión de hipogonadismo asociado a la senilidad en el varón, sin introducirnos en la terapia de reemplazo androgénico, pretende ser un aporte para avanzar en un cambio de actitud del médico frente a este problema.

\section{Bases fisiológicas y clínicas}

La testosterona secretada por las células de Leydig testiculares durante la vida fetal determina la diferenciación y el desarrollo del aparato genital y organiza las estructuras neurológicas base del dimorfismo sexual cerebral $(1,2)$. Mantiene altos niveles hasta los primeros meses de recién nacido para disminuir posteriormente. Aumenta al inicio de la pubertad, activando estructuras previamente organizadas, vinculadas a líbido y erección a nivel hipotálamico y límbico; otros órganos blancos son piel, músculo, hígado, 
médula ósea, hueso y órganos genitales masculinos. Su acción se ejerce en el órgano blanco en interacción con otros factores, por ejemplo en las zonas cerebrales sensibles a testosterona con factores emocionales, socioculturales etc., para determinar la conducta sexual; en el hueso y músculo con el eje hormona de crecimiento IGF 1.

La secreción de testosterona resulta del funcionamiento del eje hipotálamo-hipófisis-testicular (HHT) constituido por neuronas productoras del factor liberador de gonadotrofinas (GNRH), gonadotropos hipofisiarios productores de $\mathrm{FSH}$ y $\mathrm{LH}$, y por los compartimentos testiculares: tubular (espermatogénesis y células de Sértoli) e intersticial: células de Leydig (testosterona). El GNRH hipotalámico se libera en forma pulsátil, alcanzando un nivel (amplitud de pulso), que a intervalos de tiempo (frecuencia de pulso), es entregado a los capilares hipotalámicos punto de partida del sistema porta hipofisiario. El GNRH está regulado por neurotransmisores, neuropéptidos y hormonas, unos estimulantes y otros inhibitorios. Los gonadotropos estimulados por GNRH son modulados por hormonas esteroidales por ejemplo LH por testosterona y FSH por las no esteroidales (inhibina, etc.).

La pulsatilidad de LH estimula la secreción de testosterona, la que a nivel sanguíneo es transportada en un $60 \%$ por una globulina (SHBG) cuya unión es poco disociable, un $38 \%$ ligada a albúmina fácilmente disociable y un $2 \%$ circula en forma libre.

El estudio hormonal del eje HHT comprende la medición basal de FSH, LH, testosterona total y libre; se puede complementar con pruebas funcionales de estimulación de los compartimentos hipotalámico (citrato de clomifeno), hipofisiario (GNRH) y testicular (gonadotrofina coriónica de acción similar a LH), mientras que el espermiograma nos permite evaluar la función tubular.

Si la testosterona basal está bajo lo normal se diagnostica hipogonadismo; si cursa con $\mathrm{LH}$ aumentada es un hipogonadismo hipergonadotropo o falla testicular primaria; si lo hace con LH baja o, inapropiadamente normal para el valor disminuido de testosterona, se trata de un hipogonadísmo hipogonadotropo por alteración hipotalámica o hipofisiaria (3).

Los hipogonadismos pueden ser congénitos o adquiridos, éstos últimos prepuberales o post puberales de inicio en la juventud, adulto joven o mayor.

\section{Hipogonadismo y envejecimiento}

Desde antiguo se asoció envejecimiento y declinación de la función testicular, homologándose a lo que ocurre en el sexo femenino con la función ovárica, aunque se reconoce históricamente que hombres de avanzada edad mantenían su vida sexual y capacidad reproductiva.

En hombres envejecidos sanos puede aparecer un hipogonadismo de inicio tardío que se ha asociado al envejecimiento; Werner en 1939 lo denominó climaterio masculino. Este cuadro comprende los síntomas y signos de lo que hoy llamamos andropausia o ADAM (androgen decline aging male) descritos en la Tabla I. Con métodos de estudios de la época (biopsia testicular, gonadotrofinas hipofisiarias por método biológico) se clasificó como hipogonadismo hipergonadotropo (4).

Este concepto se mantuvo sin mayor avance hasta 1970 en que al poder medirse testosterona y LH plasmáticas por método de radioinmunoensayo, se desarrollaron estudios de la relación entre

Tabla I

\section{SÍNTOMAS Y SIGNOS DE HIPOGONADISMO MASCULINO ASOCIADO AL ENVEJECIMIENTO}

\section{Síntomas neurológicos y vasomotores:}

- Bochornos similares a aquellos del climaterio femenino

- Episodios de sudoración

- Insomnio

- Nerviosismo

Funciones cognitivas y alteraciones del humor:

- Irritabilidad y letargo

- Disminución de la sensación de bienestar

- Pérdida de motivación

- Dificultades con la memoria reciente

- Síntomas depresivos

- Baja autoestima

Masculinidad / Virilidad:

- Disminución de energía física

- Disminución de fuerza y masa muscular

- Pérdida de vello corporal sexual

- Obesidad abdominal

Sexualidad:

- Disminución del interés o deseo sexual

- Reducción de la actividad sexual

- Pobre función eréctil

- Baja calidad del orgasmo

- Menor volumen del eyaculado 
función testicular y edad. Entre 1970 e inicios de los 80 , diversos autores en estudios de cortes transversales de varones senescentes encontraron resultados contradictorios: 1.- descenso de la testosterona en la mayoría de los evaluados; 2.escasa diferencia con la población joven; 3.tendencia a una lenta disminución de la testosterona con la edad, con subgrupos en rango similar al joven y otros en rango hipogonádico $(5,6,7)$ Estos hechos hacían dudar a unos en la existencia de este cuadro, ¿andropausia : realidad o ficción? y otros creían que afectaba a la generalidad de los hombres (8).

El análisis crítico de estos trabajos señaló: 1.mala selección de pacientes al conformar los grupos a estudiar. Al ser reclutados en servicios geriátricos, hogares de ancianos u hospitalizados, no se consideraron las condiciones generales de salud, aspectos psicosociales, enfermedades sistémicas y uso de fármacos, condiciones que podían determinar descenso de la testosterona. Otros grupos no eran representativos de la población general de los hombres mayores, ya que eran excepcionalmente sanos, activos laboral, social y sexualmente, lo que influía en que la testosterona y espermiograma no se diferenciaban grandemente de los jóvenes (7). 2.- Errores metodológicos en el estudio de laboratorio. Los autores que no encontraron diferencias entre jóvenes y mayores midieron testosterona en las primeras horas de la tarde, sin considerar su ritmo circadiano que determina un valor matutino 25 a $30 \%$ superior al de la tarde. Este ritmo se pierde con el envejecimiento determinando valores matutinos de testosterona menores en hombres mayores versus jóvenes, diferencia que no se aprecia al medirla en la tarde (9).

La investigación entre testosterona y envejecimiento avanzó al disponerse de exámenes de laboratorio que medían testosterona libre y la proteína transportadora, como también al dilucidarse si en hombres sanos, el consumo de alcohol, estilo de vida y actividad física podían influir igual o más que la edad en los niveles de testosterona. Estudios realizados en monjes por Vermeulen (10) y posteriormente el estudio epidemiológico de Rancho Bernardo (11) demostraron que la edad era el factor determinante en la disminución progresiva de la testosterona.

La medición de testosterona total no nos informa sobre la testosterona biologicamente activa que es aquella que está fácilmente disponible para los tejidos efectores. La testosterona biodisponible o biológicamente activa comprende la testosterona libre y la ligada a albúmina. Con la edad aumenta la SHBG y disminuye la testosterona libre y por lo tanto la actividad biológica o acción de la testosterona en los efectores (12).

La sensibilidad para diagnosticar hipogonadismo asociado al envejecimiento, mejora notablemente si además de la testosterona total, es medida la libre, o mejor aún, la biodisponible.

Hasta los primeros años de la década de los 80 se había considerado el hipogonadismo asociado al envejecimiento o climaterio masculino, como una falla testicular primaria (insuficiencia Leydigiana) o hipogonadismo hipergonadotropo, y así aparecía clasificado en los textos de la época (13). Histológicamente se demostró atrofia testicular tanto tubular como intersticial, cuya etiología fue atribuida por autores japoneses a un menor flujo sanguíneo por ateroesclerosis (14). El aumento de FSH y LH se debe a un menor feedback negativo por disminución de inhibina de las células de Sértoli tubulares, y de testosterona por un menor número y funcionalidad Leydigiana (15). Sin embargo, no todos los autores encontraron una elevación patológica de FSH y LH, lo que puso en discusión que la base de este cuadro residiera solamente en una insuficiencia Leydigiana. El estudio longitudinal de Morley, demostró en 15 años de seguimiento, sólo un $10 \%$ de aumento de LH pese a la disminución promedio de testosterona total de 110 ng/dl cada 10 años (16).

Existiendo una falla Leydigiana asociada al envejecimiento y disminuyendo la testosterona, un hipotálamo e hipófisis normales deberían producir un alza de LH, lo que ocurre en pocos casos. Winter y Troen plantearon la coexistencia de falla primaria testicular y una alteración neuroendocrina hipotálamo hipofisiaria que impide el alza de LH que debería producirse por la falla primaria testicular (17).

Los factores testiculares e hipotalámico hipofisiarios involucrados en hipogonadismo del envejecimiento se esquematizan en la Tabla II.

La alteración de la pulsatilidad de LH podría deberse a una falla del generador de pulsos del GNRH hipotalámico o a una alteración hipofisiaria. El compromiso neuroendocrino hipotalámico responsable de esta alteración funcional, se debería a envejecimiento de las neuronas secretoras de GNRH o alteración de la regulación que ejercen neuropéptidos y neurotransmisores, materia abierta a la investigación $(18,19)$. La prueba de estímulo hipotalámico con citrato de clomifeno, ha demostrado una menor respuesta de LH y testosterona en hombres mayores comparados con jóvenes (20). 


\section{Tabla II}

\section{FACTORES TESTICULARES E HIPOTALÁMICO HIPOFISIARIOS INVOLUCRADOS EN HIPOGONADISMO MASCULINO ASOCIADO AL ENVEJECIMIENTO}

1.- Factor testicular:

- Disminución de la cantidad de células de Leydig

- Disminución de la respuesta de las células de Leydig a la gonadotrofina coriónica

- Alteraciones de enzimas de la esteroidogénesis

- Alteraciones de la circulación arterial del testículo

2.- Factor hipotálamo-hipofisiario:

- Pérdida del ritmo circadiano de testosterona

- Disminución de frecuencia y amplitud de pulsos de LH

- Disminución de la bioactividad de LH

- Disminución del umbral hipotálamo hipofisiario a la acción frenadora de los andrógenos

En resumen, el hipogonadismo asociado al envejecimiento se puede presentar con exámenes de laboratorio que indiquen hipogonadismo hipergonadotropo (LH alta, testosterona baja) o hipogonadotropo (LH baja o inadecuadamente normal para testosterona baja)

Desde 1990 hay consenso que la disminución paulatina, aunque no universal, de la testosterona en hombres sanos estaba asociada al envejecimiento como había anticipado Vermeulen en 1971 (5) y corroborado por el Massachusetts Male Aging Study, cuyos datos se presentan en la Tabla III, destacándose el mayor descenso de la testosterona libre que la total (21).

Existen varones que mantienen un rango normal hasta avanzada edad, y otro grupo queda bajo el nivel mínimo de testosterona del hombre joven siendo clasificado como hipogonádico. Estudios

Tabla III

\section{CAMBIOS DE LOS ESTEROIDES SEXUALES} ASOCIADOS AL ENVEJECIMIENTO MASCULINO (21)

- Descenso de la testosterona total de $0,4 \%$ al año.

- Disminución de la testosterona libre de 1,2\% anual. A los 60 años existe un $40 \%$ del nivel observado a los 25.

- $1 \%$ de descenso anual de la testosterona ligada a albúmina.

- Aumento de 1,2\% de SHBG por año.

- Disminución DHEA-S de $2 \%$ por año.

- Posible disminución de la dihidrotestosterona y sus metabolitos.

- Posible disminución del receptor de andrógenos o su afinidad. en varones de 50 a 80 años encuentran cifras variables de hipogonadismo, $11,4 \%$ en Austria (22), $19,7 \%$ en Seattle y $19,1 \%$ en Atlanta (23). Vermeulen en varones sobre 75 años diagnostica un $30 \%$ de hipogonadismo (24). Al separar los pacientes por tramos de edades, Harman encuentra $20 \%$ de hipogonadismo sobre los 60 años, 30 $\%$ sobre los 70 y $50 \%$ sobre los 80 años midiendo testosterona total (25). Basados en estudios con testosterona libre las cifras de hipogonadismo asociado al envejecimiento son de $30 \%$ sobre 60 años y $80 \%$ sobre los 80 (26).

Existe controversia si los síntomas similares al hipogonadismo del joven, se deben exclusivamente a la disminución de testosterona o a otros cambios: disminución de GH e IGF 1 (somatopausia), de dehidroepiandrosterona sulfato (adrenopausia), que también influyen en funciones mentales (ánimo y cognición), en la musculatura y tejido óseo (27).

Aún no está resuelto como caracterizar objetivamente el espectro clínico del déficit de andrógenos del hombre mayor; cuestionarios como el de San Luis (Tabla IV) (28) o el AMS (aging male symptoms) (29), han tratado de medir objetivamente los síntomas y relacionarlos con los niveles de andrógenos. En el primero de estos trabajos realizados en hombres entre 40 y 62 años, se encontró correlación entre síntomas y niveles de testosterona libre y biodisponible, hecho no observado en el segundo que comprendía varones entre 74 y 89 años; de acuerdo a estos resultados no podemos aún tener la seguridad que un score elevado de síntomas pueda predecir el nivel de andrógenos plasmáticos.

Los síntomas de hipogonadismo similares a los del hombre joven, que en el hombre mayor coexisten con nivel de testosterona baja podrían deberse más al hipogonadismo que a enveje-

\section{Tabla IV}

\section{CUESTIONARIO DE SAN LUIS (28)}

- ¿Tiene disminución de la libido?

- ¿Tiene pérdida de energía?

- ¿Tiene disminución de fuerzas y/o duración?

- ¿Ha perdido altura?

- ¿Ha dejado de gozar la vida?

- ¿Está Ud triste o regañón?

- ¿Tiene erecciones menos vigorosas?

- ¿Ha experimentado deterioro reciente en su capacidad para hacer deportes?

- ¿Tiene que dormir después de comer?

- ¿Ha experimentado deterioro reciente en su capacidad laboral? 
cimiento; los que ocurren con testosterona normal podrían ser expresión de envejecimiento y/o alteración de otros ejes endocrinos. No puede excluirse que un nivel normal bajo de testosterona, sea biológicamente inadecuado para ese paciente, colocándolo en una deficiencia relativa frente a valores más altos de otra época de su vida.

Dentro de los síntomas del hombre mayor destacan disminución de líbido y mayor frecuencia de disfunción eréctil, ambos influidos por muchas variables como salud general, enfermedades sistémicas, fármacos, situación psicosocial y de pareja, etc., que deben ser considerados antes de responsabilizar exclusivamente a la testosterona.

La testosterona estimula la conducta sexual en hombres y machos de otras especies, y la terapia de sustitución en el varón hipogonádico joven aumenta la libido, las erecciones nocturnas y espontáneas, experimentando una notable mejoría en su vida sexual.

No hay que confundir con patológicos, los cambios fisiológicos que presenta el hombre al avanzar en edad: disminución de la frecuencia y duración de la erección nocturna, menor frecuencia coital, aumento del tiempo entre estímulo e inicio de la erección, prolongación del tiempo para lograr orgasmo y eyaculación, menor intensidad del orgasmo y aumento del período refractario post orgásmico (30).

Estudios en hombres mayores contradicen la inadecuada práctica médica de no interesarse por la función sexual en esta época de la vida, ya que como señaló Kinsey (31) en sus encuestas sobre conducta sexual del hombre norteamericano realizadas en 1948, había una disminución de la frecuencia coital con la edad aunque un subgrupo de individuos mayores de 80 años mantenían su vida sexual. Davinson en 1983 llegó a similar conclusión (32). Morley encuentra que sobre los 60 años, el $70 \%$ de los varones son sexualmente activos y en un grupo de 80 años muy sano, el 30 $\%$ mantenía actividad coital semanal (33). El Massachusetts Aging Study, estudio epidemiológico realizado en una población de varones entre 40 y 70 años, demostró algún grado de disfunción eréctil en el $52 \%$, existiendo falla completa de un 5 $\%$ a los 40 años y de un $15 \%$ a los 70 (31). El estudio longitudinal de envejecimiento de Baltimore demostró en hombres sanos un $8 \%$ de disfunción eréctil a los 55 años, $25 \%$ a los 65 años, $55 \%$ a los 75 años y $75 \%$ a los 80 años (15).

La función eréctil depende de factores psicológicos, neurológicos, vasculares, adecuada función endotelial, liberación de neurotransmisores (óxido nítrico) en un cuerpo cavernoso cuyos tejidos sean sanos, por lo cual la testosterona no juega un rol exclusivo (31). En los pacientes con testosterona baja debe evaluarse integralmente la función eréctil, ya que el hipogonadísmo puede ser concomitante con ella y no necesariamente la causa de la disfunción (34).

Se discute el efecto de la edad en la fertilidad, ya que en 1950 se señaló que con el envejecimiento se alteraba la motilidad y morfología de los espermatozoides, sin embargo la Biblia relata la posibilidad de engendrar hijos a edad avanzada y en medios de comunicación igual hecho se ha señalado en figuras del arte, cine, etc. (35). Nieschlag en 1982 demostró que varones sanos envejecidos comparados con padres jóvenes, tenían poca diferencia en espermiograma y función espermática (7). Tal como ocurre con la testosterona, habrían varones cuya fertilidad disminuye y en otros en que se mantiene, requiriéndose más tiempo para lograr embarazo y existiendo mayor probabilidad de alteraciones cromosómicas (36).

Podemos concluir que en todos los varones envejecidos hay un descenso promedio de sus niveles de andrógenos, existiendo una amplia variabilidad en su cuantía; sólo entre un 20 a $30 \%$ presentan valores bajo el límite inferior de testosterona total y libre del hombre joven, diagnosticándose en ese caso un hipogonadismo, cifra que aumenta después de los 70 años (26). Este hipogonadismo asociado al envejecimiento se denomina en la actualidad andropausia o ADAM. El diagnóstico de este cuadro clínico se basa en historia clínica y examen físico completo. Como en toda hipotestosteronemia debe realizarse más de un examen para confirmarla y para afirmar que se debe al envejecimiento se deben excluir otras patologías que afecten al eje HHT.

\section{Problemas pendientes}

1.- Utilizar una definición semánticamente y biológicamente adecuada.

No puede homologarse el cese de la función ovárica con la testicular, no siendo aplicables los términos utilizados en la mujer como menopausia y climaterio para el varón. Menopausia significa cese de la menstruación, y climaterio significa escalón o etapa definida de la vida en que ocurre al cese completo de la función gonadal. Se ha estimado que andropausia es un mal nombre para una verdadera entidad clínica ya que pausia significa cese o fin de la masculinidad (andros), término poco feliz para la autoestima del varón, ya 
que por presentar síntomas de envejecimiento y un nivel disminuido de testosterona, el hombre no deja de ser plenamente hombre. Posteriormente se intentó reemplazar andropausia por la sigla PADAM ( partial androgen deficiency of aging male), en que la palabra parcial no explicaba claramente si se refería a que afectaba sólo a parte de los hombres, o podía ser entendida como una deficiencia relativa frente a niveles previos de testosterona en otras etapas de la vida, por lo cual el último término en boga es ADAM (androgen decline in the aging male) o hipogonadismo asociado al envejecimiento; es deseable que todos usemos una misma nomenclatura para referirnos al mismo problema $(37,38)$.

2.- Debe encontrarse un patrón de oro para el diagnóstico clínico y bioquímico de ADAM, mediante cuestionario y un punto de corte en el nivel de andrógenos, que permitiera separar normalidad de deficiencia, y válido para cualquier tramo de edad del hombre mayor.

3.- A nivel de laboratorio debe contarse con un ensayo que mida la testosterona biodisponible de fácil ejecución y costo razonable; se sugiere por ahora utilizar la formula propuesta por Vermeulen para la estimación de testosterona libre (39).

4.- Se requiere mayor experiencia en base a seguimientos longitudinales de gran número de pacientes, para aclarar la correlación entre síntomas atribuibles a hipogonadismo y niveles de andrógenos.

5.- Mejorar nuestros conocimientos en el hombre envejecido sobre acción de la testosterona a nivel óseo, muscular, cognitivo y sexual, para tener datos objetivos que sustenten la terapia de reemplazo androgénico en este hipogonadismo.

6.- Previo a generalizar el reemplazo androgénico, los estudios deben demostrar eficacia y seguridad de la terapia.

\section{CONCLUSIONES}

¿Qué hacer actualmente frente a un paciente con hipogonadismo del envejecimiento? Por ahora los especialistas podrían realizar sustitución androgénica en el grupo hipogonádico en ausencia de contraindicaciones (próstata, etc.) y con un control estricto de su seguridad.

En pacientes con síntomas sugerentes de hipogonadismo y niveles normales bajos de testosterona, es posible que su umbral androgénico sea mayor, existiendo una deficiencia relativa de testosterona entre el nivel actual y valores mayores de otros períodos de su vida; en este caso se podría intentar una prueba terapéutica con testosterona. Un análisis más objetivo de este punto se lograría si el médico solicitara determinaciones de testosterona en adultos jóvenes asintomáticos y recomendara conservar los resultados para comparaciones futuras.

Se requiere prudencia antes de generalizar la terapia de reemplazo androgénico en el varón envejecido, posiblemente sólo un grupo la necesite y es necesario conocimiento especializado para indicarla.

La historia reciente del debate respecto a terapia de reemplazo estrogénico en el climaterio, nos enseña que no podemos tropezar dos veces con la misma piedra en terapias de reemplazo hormonal con esteroides sexuales.

El déficit de una hormona no explica todo el proceso de envejecimiento y difícilmente su administración será la gran panacea. El entusiasmo terapéutico no puede hacernos ignorar riesgos versus beneficios, y la necesidad de información, terapia consensuada con el paciente y su adecuado control.

\section{BIBLIOGRAFÍA}

1. Devoto E. Aspectos psicoendocrinos de la conducta sexual. En: Jadresic A, Ojeda C, Pérez G, ed. Psiconeuroendocrinología. Santiago: Publicaciones Técnicas Mediterráneo, 2000; 51 - 63.

2. Gorski RA. Androgen: effects on the brain. Cognitive, sexual and aggressive behavior, an overview. In: Bhasin S, Gabelnick H, Spieler J, Swerdloff R, Wang $\mathrm{C}$, Kelly Ch, ed. Pharmacology, Biology, and Clinical Application of Androgen. New York: A John Wiley \& Sons, INC., 1996; $157-177$.

3. Weinbauer G F, Gromoll J, Simoni M, Nieschlag E. Physiology of testicular function. In: Nieschlag E, Behre HM, ed. Male reproductive health and dysfunction. Berlin: Springer- Verlag, 1997; 25 - 57

4. Werner AA. The male climateric, report of 54 cases. JAMA 1945; 127: 705.

5. Vermeulen A, Rubens R, Verdonck L. Testosterone secretion and metabolism in male senescense. J Clin Endocrinol Metab 1972; 34: 730 - 735.

6. Stearns EL, Mc Donnell JA, Kaufman BJ, Lucman TS, Winter JSD, Faiman C. Declining testicular function with age. Am J Med 1974; 57: 761 - 766.

7. Nieschlag E, Lammers U, Freischem CW, Langer K, Wickings EJ. Reproductive functions in young fathers and grandfathers. J Clin Endocrinol Metab 1982; 55: $676-681$

8. Harman SM, Blackman MR. Male menopause, myth or menace? Endocrinologist 1994; 4: 212 - 217.

9. Bremner WJ, Vitiello MV, Prinz PN. Loss of circadian rhythmicity in blood testosterone levels with aging 
in normal men. J Clin Endocrinol Metab 1983; 56: $1278-1281$.

10. Vermeulen A, Deslypere J P. Leydig cell function in normal men: effect of age, life - style, residence, diet and activity. J Clin Endocrinol Metab 1984; 59: 955 - 962.

11. Ferrini RL, Barret-Connor E. Sex hormones and age: a cross - sectional study of testosterone and estradiol and their bioavailable fractions in community-dwelling men. Am J Epidemiol 1998; 147: $750-754$.

12. Nankin HR, Calkins JH. Decreased bioavailable testosterone in aging normal and impotent men. J Clin Endocrinol Metab 1986; 63: 1418 - 1420.

13. Griffin JE, Wilson JD. Trastornos de los testículos y del aparato reproductor masculino. En: Wilson JD, Foster DW, ed. Endocrinología. Buenos Aires: Editorial Médica Panamericana, 1985; 389 - 463.

14. Nieschlag E, Freischmen C W, Wickings E J, Schürmeyer Th. A new look at testicular functions in old age. In D'Agata R, Lipsett M B, Polosa P, Van der Molen $\mathrm{H} J$, ed. Recent advances in male reproduction: molecular basis and clinical implications. New York: Raven Press, 1983;145 - 149.

15. Harman SM, Tsitouras PD. Reproductive hormones in aging men. I. Measurement of sex steroids, basal luteinizing hormone and Leydig cell response to human chrorionic gonadotropin. J Clin Endocrinol Metab 1980; 51 : 35 - 40.

16. Morley JE, Kaiser FE, Perry HM, Patrick P, Morley PMK, Stauber PM et al. Longitudinal changes in testosterone, luteinizing hormone and folliclestimulating hormone in healthy older men. Metabolism 1997; 46: 410 - 413.

17. Winters SJ, Troen P. Episodic luteinizing hormone (LH) secretion and the response of $\mathrm{LH}$ and folliclestimulating hormone to $\mathrm{LH}$-releasing hormone in aged men: evidence for coexistent primary testicular insufficiency and an impairment in gonadotropin secretion. J Clin Endocrinol Metab 1982; 55: $560-565$.

18. Kaufman JM, Deslypere JP, Giri M, Vermeulen A. Neuroendocrine regulation of pulsatile luteinizing hormone secretion in elderly men. J Steroid Biochem Molec Biol 1990; 37: 421 - 430.

19. Katznelson L. Neuroendocrine aspects of testosterone insufficiency with aging. Endocrinologist 1999; 9: 190 - 196.

20. Tenover J, Matsumoto AM, Plymate SR, Bremmer WJ. The effects of aging in normal men on bioavailable testosterone and luteinizing hormone secretion: response to clomiphene citrate. J Clin Endocrinol Metab 1987; 65: 1118 - 1126.

21. Gray A, Feldman HA, McKinlay JB, Longcope C. Age, disease and changing sex hormone levels in middle-age men: results of the Massachusetts male aging study. J Clin Endocrinol Metab 1991; 73: $1016-1025$.

22. Lunglmayr $\mathrm{G}$. Trial on androgen supplementation in aging man. In: Waites G F H, Frick J, Baker G W H, ed. Current Advances in Andrology. Bologna: Moduzzi Editore, 1997; 289 - 292.

23. Tenover J. Androgen therapy in aging men. In: Bhasin S, Gabelnick H L, Spieler J M, Swerdloff R S, Wang C, Kelly C, ed. Pharmacology, biology, and clinical applications of androgens. New York: Wiley - Liss, 1996; $309-318$.

24. Vermeulen A. Androgen replacement therapy in the aging male. A critical evaluation. J Clin Endocrinol Metab 2001; 86: 2380 - 2390.

25. Harman SM, Metter EJ, Tobin JD, Pearson J, Blackman MR. Longitudinal effects of aging on serum total and free testosterone levels in healthy men. J Clin Endocrinol Metab 2001; 86: 724 - 731.

26. Tenover J. Declining testicular function in aging men. Int J Impot Res 2003; 15: 3 - 8.

27. Tenover J. Risks and benefits of testosterone in older men. In: Robaire B, Chemes H, Morales C R, ed. Andrology in the 21st Century. Englewood: Medimond Medical Publication, 2001; 395 - 405.

28. Morley JE, Charlton E, Patrick P, Kaiser FE, Cadeau $P$, McCready $D$ et al. Validation of a screening questionnaire for androgen deficiency in aging males. Metabolism 2000; 49: 1239 - 1242.

29. T'Sjoen G, Goemaere S, De Meyere M, Kaufman JM. Perception of males'aging symptoms, health and well-being in elderly community-dwelling men is not related to circulating androgen levels. Psychoneuroendocrinol 2004; 29: 201 - 214.

30. Tsitouras PD. Effects of age on testicular function. Endocrin Metab Clin North Am 1987; 16: 1045 - 1059.

31. Kinsey AC, Pomeroy WB, Martin CE. Sexual behavior in the human male. Philadelphia: W B Saunders, 1948; $236-237$.

32. Davidson JM, Chen JJ, Crapo L, Gray GD, Greenleaf WJ, Catania JA. Hormonal changes and sexual function in aging men. J Clin Endocrinol Metab 1983; 57: $71-77$.

33. Morley JE, Kaiser FE. Sexual function with advancing age. Med Clin North Am 1989; 73: 1483 - 1495.

34. Jorenman SG, Morley JE, Mooradian AD, Davis SS, Kaiser FE, Silver $\mathrm{J}$ et al. Secondary hypogonadism in older men: its relation to impotence. J Clin Endocrinol Metab 1990; 71: 963 - 969.

35. Tsitouras PD, Bulat $\mathrm{T}$. The aging male reproductive system. Endocrin Metab Clin North Am 1995; 24: $297-315$.

36. Meacham RB, Murray MJ. Reproductive function in the aging male. Urol Clin North Am 1994; 21: 549 - 556.

37. Gooren LJG. The age-related decline of androgen levels in men: clinically significant? British J Urology 1996; 78: 763-768.

38. Morales A, Heaton JPW, Carson CC. Andropause: a misnomer for a true clinical entity. J Urol 2000; $705-712$.

39. Vermeulen A, Verdonck L, Kaufman JM. A critical evaluation of simple methods for the estimation of free testosterone in serum. J Clin Endocrinol Metab 1999; 84: 3666 - 3672. 